\title{
The relationship between COVID-19 and the dental damage stage determined by radiological examination
}

\author{
Dursun Ali $\operatorname{Sirin}^{1}\left[\right.$ [D Fatih Ozcelik ${ }^{2}$
}

Received: 23 October 2020 / Accepted: 23 November 2020 / Published online: 3 January 2021

(c) Japanese Society for Oral and Maxillofacial Radiology and Springer Nature Singapore Pte Ltd. 2021, corrected publication 2021

\begin{abstract}
Objective COVID-19 causes severe complications particularly in respiratory system especially in the elderly and those with poor general health. General and oral health are closely related to the course of such viral diseases. This study aims to reveal the relationship between the Dental Damage Stage (DD Stg) and the severity of COVID-19 disease.

Materials and methods The study included 137 patients (20-65 years) based on oral examination records and panoramic X-rays of 1516 COVID-19 patients diagnosed through real time PCR tests. DD Stg were determined according to data of Apical Periodontitis Grading Scale, Radiologic Alveolar Bone Loss and Pathophysiological Process of Dental Caries obtained from dental radiological images. DD Stg were determined according to the severity of dental pathology and compared in terms of age, gender, numbers of dental caries (NDC), dental implants (NDI), root canal treatment (NRCT), tooth fillings (NTF), missing teeth (NMT) and hospitalization due to COVID-19 (NHC), presence of chronic disease (CD), and symptom associated with COVID-19 (SAC)

Results Patients in DD Stg 3 had significantly higher age and mortality. CD, NDC and NHC values were higher in DD Stg 2 and 3 than in DD Stg 0 and 1. NHCs were higher in DD Stg 3 than in DD Stg 2. NMTs were higher in DD Stg 3 than other stages. SACs were significantly lower in DD Stg 0 than in DD Stg 1, 2 and 3. NDC, NHC, SAC and CD were effective on DD staging; they were moderately positively related.
\end{abstract}

Conclusions The relationship detected between DD stage and the severity and prognosis of viral disease such as COVID-19 was found to be remerkable for extensive studies.

Keywords Dental damage stage · COVID-19 $\cdot$ Hospitalization $\cdot$ Dental health

\section{Introduction}

COVID-19 disease, which has come to the fore with the occurrence of pneumonia cases with unknown etiology towards the end of 2019 in Wuhan, China, has caused a large number of deaths by creating a severe pandemic across the world. Strong immune system, healthy lifestyle,

Dursun Ali Sirin

dtalisirin@gmail.com

1 University of Health Sciences, Hamidiye Faculty of Dentistry, Department of Endodontics and Sultan 2. Abdulhamid Han Training Hospital, Saglik Bilimleri Universitesi, 34668 Istanbul, Turkey

2 University of Health Sciences, Sultan Abdülhamid Han Training Hospital, Biochemistry Specialist Department of Medical Biochemistry, Sağlık Bilimleri Üniversitesi, İstanbul, Turkey and physiological and psychological health are among the important factors in combating this disease [1-3]. These factors are closely related to oral and dental health. Because a strong immune system and all biochemical and physiological processes are possible with correct and balanced nutrition. Oral and dental health is directly associated with nutrition, therefore, is the first barrier against a wide variety of viruses including coronavirus. For this reason, many parameters, which are indicators of oral and dental health status, provide us with important data in combating diseases. As it can be understood from here, oral and dental health status, which is one of the most important indicators of human health, has a key role in the fight against many viral and bacterial diseases $[4,5]$.

There are several scales and classifications that are used as indicators of dental and periodontal health status. One of them, the Periapical Index (PAI), was developed by Ørstavik et al. in 1986 and has been used for the classification of 
apical periodontitis (AP) for many years [6]. The PAI scoring system numbers the teeth for AP from 1 to 5 according to radiolucency of the periapex on radiographs. The Apical Periodontitis Grading Scale (APGS), which was developed by Sirin et al. based on PAI, is a new scale used to that classifies the AP according to the number of affected teeth and the severity of AP and has been used in several studies [7, 8]. According to the APGS, patients with PAI score 1 and 2 are categorized as AP Grade 0 , those with only one tooth included AP with a PAI score of 3 or 4 are considered AP Grade 1, those with more than one tooth included AP with a PAI score of 3 or 4 are classified as AP Grade 2, and those with at least one tooth included AP scored as a PAI 5 are described as AP Grade 3.

The classification of periodontal status has been modified in recent years according to new scientific evidence [9]. In 2017, the American Academy of Periodontology and the European Federation of Periodontology developed a new multidimensional staging and grading system and recommended its use in the classification of periodontitis [10]. Periodontal status can be evaluated clinically by measuring clinical attachment loss (CAL), probing pocket depth (PPD) and gingival recession (GR) [11]. In addition to these, radiographic bone loss (RBL), which evaluates alveolar bone loss in millimeters, and \% RBL classification, which evaluates bone loss in percentage, have come to the fore in the evaluation of periodontal status, providing some useful information oral and dental health. RBL measurement is performed by evaluating the quantitative and qualitative perspectives of imaginable interproximal bone, which includes the distance between the cement-enamel junction (CEJ) and the most coronal level, where the periodontal ligament interval is considered to have a normal width [12]. Both RBL and \%RBL classifications are related to the loss of bone tissue the teeth are connected, resulting in spontaneous loss of the teeth in the future $[10,13]$. All these classifications have emerged from an effort to find an accurate indicator of the oral and dental health status. Cariogenic bacteria, susceptibility of the tooth surface, and nutritional effects are blamed for the pathophysiological mechanisms of the onset and progression of dental caries $[14,15]$. Thus, the pathophysiological process of dental caries was called the pathophysiological process of dental caries (PPDC).

In the light of all this information, this study has developed a new scoring called Dental Damage Stage (DD Stg) and based on APGS, RBL and PPDC, as a new oral and dental status indicator that can be directly or indirectly related to the course of viral diseases including COVID-19. Then the study has examined the relationship between the DD Stg, the number of dental caries (NDC), the number of dental implants (NDI), the number of root canal treatment (NRCT), the number of tooth fillings (NTF), the number of missing teeth (NMT), the number of crown (NC), the number of hospitalizations due to COVID-19 (NHC), the symptom associated with COVID-19 (SAC), the presence of chronic disease (CD), and the rate of mortality due to COVID-19.

\section{Materials and methods}

This analytical retrospective study was conducted in the University of Health Sciences, Sultan 2. Abdulhamid Han Training and Research Hospital Dental Services in Turkey using patient records between March 10 and July 15, 2020. Patients who had oral and dental health records and a history of COVID-19 were included in the study. Those without oral and dental health records were excluded from the study. Ethical approval was obtained from the University of Health Sciences, Hamidiye Clinical Research Ethics Committee (registration number: 20/180). Furthermore, this study was conducted with the approval (No. 2020-05-27T12-20-31) of the T.R Ministry of Health Research Platform.

\section{Study sample and design}

The study examined patients among 1,516 patients with positive real time PCR COVID-19 test whose examination and treatment for COVID-19 were performed in our hospital. A total of 137 patients aged 20-65 years, including 71 males and 66 females, who referred to our dental outpatient clinic for dental examination, treatment or control in the last 6 months and whose oral examination records and panoramic X-rays were available in the system were included in the study. In addition, all patients' $\mathrm{ABO}$ blood group and Rhesus (Rh) factor test results were evaluated.

\section{Dental radiographs}

Medical and dental radiographic information of the COVID19 patients was collected using the Nucleus Medical Information System (Monad software, Istanbul, Turkey), a hospital information and automation system. An experienced endodontist evaluated their panoramic radiographs retrospectively twice at different times. The radiographs were downloaded from the Extreme Pacs version 4.3 (Ankara, Turkey), the official medical image and archiving system, and were produced in a dim room using contrast, density, zoom and measurement settings on a 20 -inch wide LCD TFT monitor (HP, Houston, USA).

The number of dental caries (NDC), the number of root canal treatment (NRCT), the number of tooth filling (NTF), the number of dental implant (NDI), the number of crown (NC) and the number of missing tooth (NMT) were determined for the patients using available dental radiographic images and reports. Then, their PAI scores and \% RBL measurements were found. The teeth with 
AP detected in radiographs were scored according to the APGS based on PAI (Fig. 1). The patients were divided into 4 groups (APGS group 0,1, 2 and 3 ) according to the severity of AP. These APGSP groups were scored as $0,1,3$ and 5 according to the severity of their AP. The computer-aided diagnoses (CAD) method was used for the RBL measurements $[16,17]$. Based on the patients' radiological data, the radiologic alveolar bone loss (RBL) was calculated in percentage (\%) by a computer program (ExtremePacs Client $\times 64$ Software, Ankara, Turkey) (Fig. 2). The patients were divided into 4 groups (RBL group 1, 2, 3, and 4) according to the severity of RBL,
Fig. 1 APGS scoring based on PAI on dental radiographic images. a Since PAI 3 was detected in tooth number 48 , the patient was included in the APGS group 1. b Since PAI 3 was detected in tooth number 17 and PAI 4 in teeth 36 and 37. the patient was included in the APSG group 2. c Since PAI 3 was detected in tooth number 18 and PAI 5 in teeth 36 and 46, the patient was included in the APSG group 3
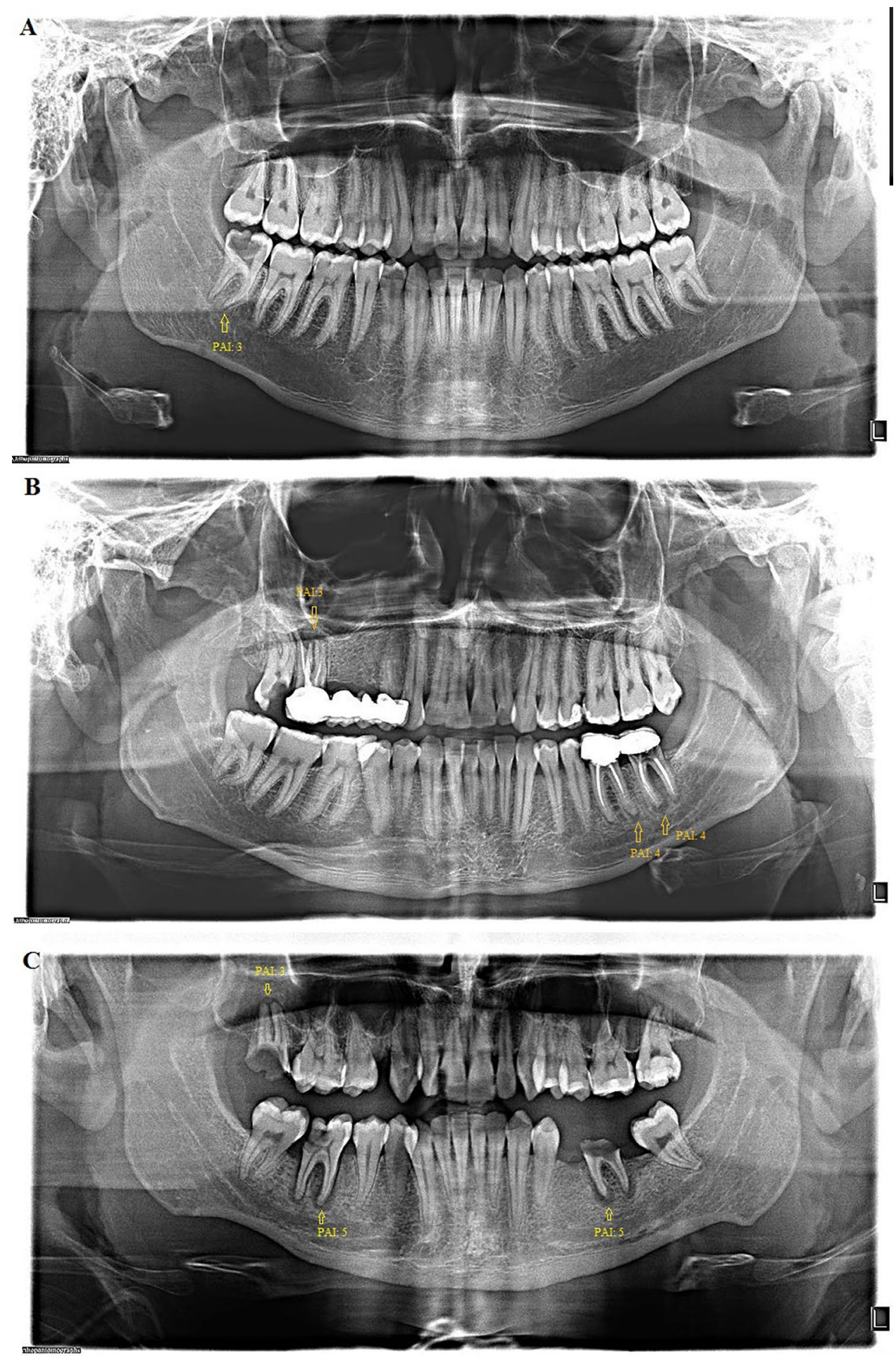


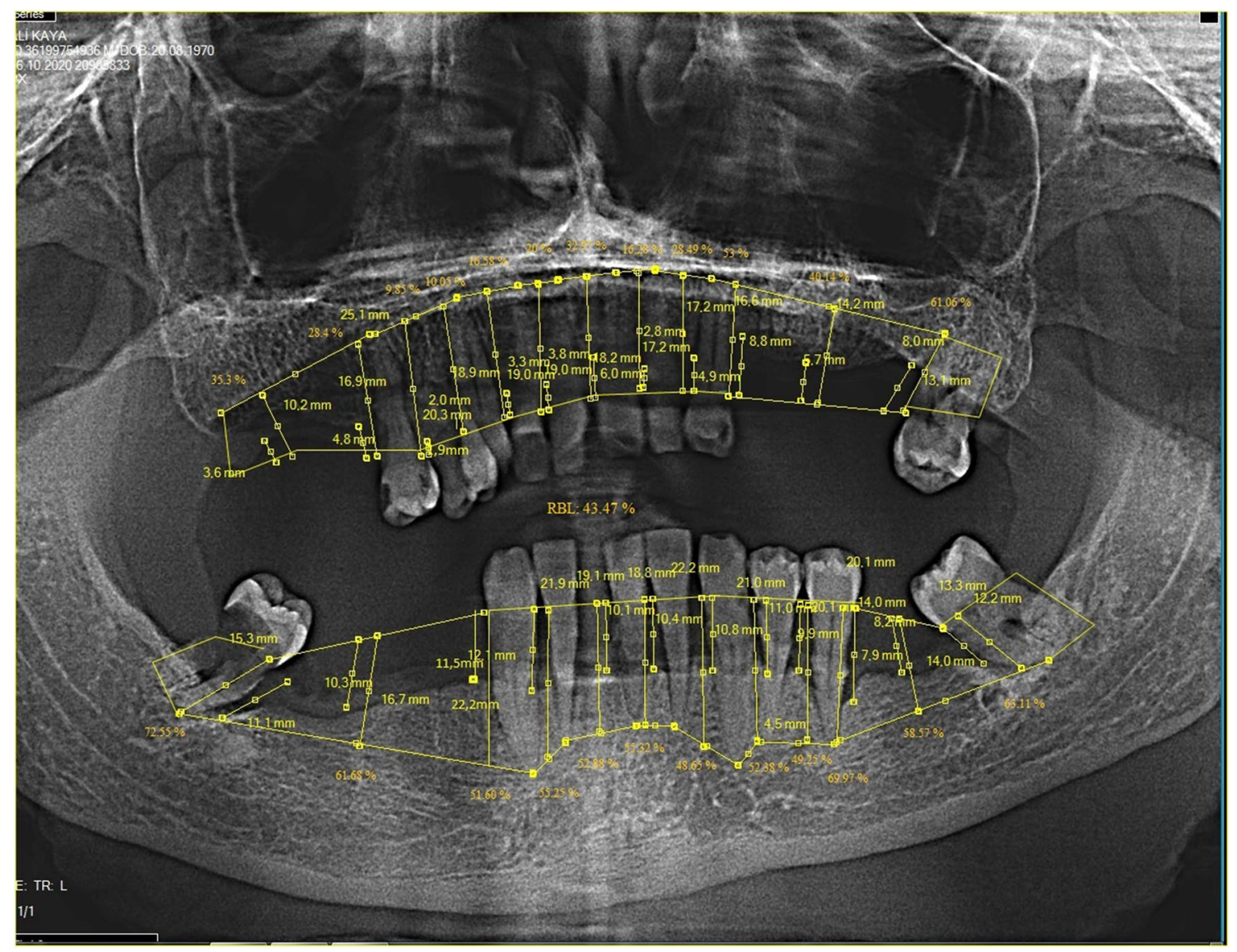

Fig. 2 RBL measurements made using computer-aided diagnosis (CAD) method. The figure shows the radiographic image of a patient whose RBL measurement was $43.47 \%$ and was included in RBL group 4

including RBL $0 \%,<15 \%, 1-33 \%$, and $>33 \%$. These RBL groups were scored as $0,1,3$ and 5 according to the severity of their RBL. The classification of PPDC was based on dental caries, where by the lack of dentine caries was classified as PPDC group 0; the presence of only one dental caries was categorized as PPDC group 1 as the first dentine caries was considered to be the beginning; and the presence of more than one dentine caries, which is an indication of dental caries progression, was considered PPDC group 2. These PPDC groups were scored as 0,1 and 3 according to their severity.

Oral and dental health status of the patients were scored based on their APGS, RBL and PPDC values. Using this scoring, their Dental Damage Stage (DD Stg) was determined as an indicator of their oral and dental health status (Fig. 3). Accordingly, those who scored 0-1 for dental health were classified in DD Stg 0 (healthy), those who scored 2-4 in DD Stg 1 (mild), those who scored 5-8 in DD Stg 2 (moderate), and those who scored 9-13 in DD Stg 3 (severe).

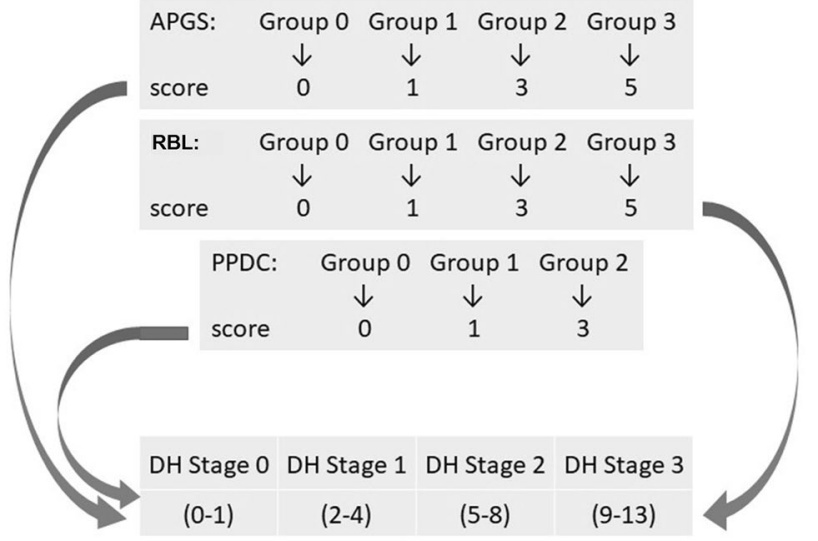

Fig. 3 Algorithm of Dental Damage Stage. Dental Damage Stage calculated on the basis of scores from APGS, RBL and PPDC. APGS Apical periodontitis grading scale, $R B L$ Radiologic alveolar bone loss, $P P D C$ Pathophysiological process of dental caries 


\section{Statistical analysis}

IBM SPSS Statistics 25 IBM (Corp, Chicago, USA) and InStat3 GraphPad Statistics Software were used for the statistical analysis of all research data. In the analysis of independent groups for more than two groups, one-way ANOVA was used to compare parametric data, and Kruskal-Wallis to compare nonparametric data. Since the study showed parametric distribution, Pearson correlation analysis was used for correlational studies of the data, and Spearman correlation analysis was used for nonparametric data. A multiple regression analysis was performed to determine the effects of a number of independent variables, including NDC, NDI, NRCT, NTF, NMT, NC, NHC, SAC and CD, on DD Stg (dependent variable), and to predict the dependent variable. Chi square test was used to evaluate categorical data.

\section{Results}

Table 1 presents the demographic and clinical data of COVID-19 patients included in the study in terms of dental health according to DD Stg. There was no statistically significant difference between the patients in DD Stg 0, 1 and 2 in terms of age, where those in the DD Stg 3 were significantly older than others.

In addition, there was no statistically significant difference between the patients in DD Stg 0, 1, 2 and 3 in terms of gender (Table 1). Similarly, there was insignificant difference between the groups in terms of NDI, NRCT, NTF and NC.

No statistical significant difference was found between those in the DD Stg 0 and Stg 1, and between those in the DD Stg 2 and Stg 3 in terms of CD, while there was a statistically significant difference between the others (Table 1). In addition, a statistically significant difference was found between those in the DD Stg 0 and Stg 2, between those in the DD Stg 0 and Stg 3, and between those in the DD Stg 1 and Stg 3 in terms of NDC. In summary, those in the DD Stg 2 and 3 had significantly higher CD and NDC than the other groups. In terms of NMT, only those in the DD Stg 3 was higher values than the others $(p<0.05)$.

Patients in the DD Stg 2 and 3 had significantly higher NHC than those in the DD Stg 0 and Stg 1 (Table 1). In addition, those in the DD Stg 3 had higher NHC than those in the DD Stg $2(p<0.05)$.

Patients in the DD Stg 0 had significantly lower SAC than those in the DD Stg 1, Stg 2 and Stg 3 (Table 1). Patients in the DD Stg 3 significantly higher mortality rate, whereas

Table 1 Comparison of the data according to dental damage stages belonging to all groups

\begin{tabular}{|c|c|c|c|c|c|}
\hline & Stage 0 & Stage 1 & Stage 2 & Stage 3 & $P$ value \\
\hline$N$ & 31 & 30 & 44 & 32 & - \\
\hline Age, year* & $39 \pm 12.3$ & $39 \pm 12.4$ & $44 \pm 12.9$ & $53 \pm 9.8$ & $<0.000^{\mathrm{a}}$ \\
\hline$p$ from post-test & \multicolumn{5}{|c|}{$>0.05,>0.05,<0.001,>0.05,<0.001,<0.05$} \\
\hline Gender, male (\%) & $12(39)$ & $13(43)$ & $25(57)$ & $21(66)$ & $0.1197^{\mathrm{a}}$ \\
\hline Number of dental implants, $n$ & $0.0 \pm 0.2$ & $0.0 \pm 0.0$ & $0.4 \pm 1.7$ & $0.3 \pm 1.0$ & $0.1354^{\mathrm{a}}$ \\
\hline Number of root canal treatment, $n$ & $1.0 \pm 1.2$ & $1.2 \pm 1.3$ & $1.7 \pm 1.8$ & $2.1 \pm 2.7$ & $0.1337^{\mathrm{a}}$ \\
\hline Number of tooth fillings, $n$ & $2.3 \pm 2.0$ & $3.0 \pm 2.7$ & $2.7 \pm 2.4$ & $2.8 \pm 2.7$ & $0.7986^{\mathrm{a}}$ \\
\hline Number of crown, $n$ & $1.8 \pm 4.7$ & $1.6 \pm 3.2$ & $4.1 \pm 6.9$ & $4.7 \pm 5.4$ & $0.1128^{\mathrm{a}}$ \\
\hline Chronic Disease, $n(\%)$ & $1(3)$ & $3(10)$ & $20(45)$ & $17(53)$ & $<0.0001^{\mathrm{a}}$ \\
\hline$p$ from post-test & \multicolumn{5}{|c|}{$>0.05,<0.001,<0.001,<0.01,<0.01,>0.05$} \\
\hline Number of dental caries, $n^{*}$ & $0.2 \pm 0.4$ & $1.0 \pm 1.2$ & $1.9 \pm 1.5$ & $2.5 \pm 2.0$ & $<0.0001^{\mathrm{a}}$ \\
\hline$p$ from post-test & \multicolumn{5}{|c|}{$>0.05,<0.001,<0.001,>0.05,<0.001,>0.05$} \\
\hline Number of missing teeth, $n^{*}$ & $3.9 \pm 3.2$ & $5.2 \pm 5.7$ & $6.4 \pm 5.6$ & $10.0 \pm 6.5$ & $<0.0001^{\mathrm{a}}$ \\
\hline$p$ from post-test & \multicolumn{5}{|c|}{$>0.05,>0.05,<0.001,>0.05,<0.01,<0.05$} \\
\hline Number of hospitalizations due to COVID-19, $n(\%)^{*}$ & $0(0)$ & $3(10)$ & $13(30)$ & $24(75)$ & $<0.0001^{\mathrm{a}}$ \\
\hline$p$ from post-test & \multicolumn{5}{|c|}{$>0.05,<0.05,<0.001,>0.05,<0.001,<0.001$} \\
\hline Symptom associated with COVID-19, $n(\%)^{*}$ & $11(35)$ & $25(83)$ & $39(89)$ & $31(97)$ & $<0.0001^{\mathrm{a}}$ \\
\hline$p$ from post-test & \multicolumn{5}{|c|}{$<0.001,<0.001,<0.001,>0.05,>0.05,>0.05$} \\
\hline $\mathrm{Ex}, n(\%)$ & $0(0)$ & $0(0)$ & $1(2)$ & $6(19)$ & $<0.0001^{\mathrm{a}}$ \\
\hline from post-test & \multicolumn{5}{|c|}{$>0.05,>0.05,<0.01,>0.05,<0.01,<0.001$} \\
\hline
\end{tabular}

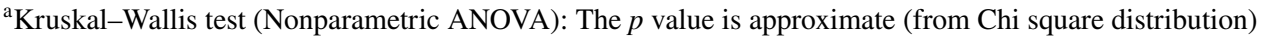

*If $p$ value obtained by ANOVA is $<0.05, p$ values of between groups (respectively, Dental Damage Stage 0-Stage1, Stage 0-Stage 2, Stage 0Stage 3, Stage 1-Stage 2, Stage 1-Stage 3 and Stage 2-Stage 3) are compared with post-test 
Table 2 Model with nine independent variables used to estimate the severity of dental damage determined by Dental Damage Stage
Table 3 Model with four independent variables used to estimate the severity of dental damage determined by Dental Damage Stage

\begin{tabular}{lclrrr}
\hline Variable & Coefficient & SE & 95\% CI & t ratio & P value \\
\hline Constant & 0.2689 & 0.1543 & $-0.029-0.566$ & 1.772 & 0.0788 \\
Number of dental caries & 0.2694 & 0.03865 & $0.194-0.345$ & 6.971 & $<0.0001$ \\
Number of dental implants & 0.06407 & 0.05858 & $-0.051-0.179$ & 1.094 & 0.2761 \\
Number of root canal treatment & 0.06030 & 0.03760 & $-0.0134-0.134$ & 1.604 & 0.1112 \\
Number of tooth filling & -0.02246 & 0.02886 & $-0.079-0.034$ & 0.778 & 0.4380 \\
Number of missing teeth & 0.01954 & 0.01388 & $-0.0077-0.047$ & 1.408 & 0.1616 \\
Number of crown & 0.01599 & 0.01326 & $-0.010-0.042$ & 1.206 & 0.2301 \\
Number of hospitalizations due to COVID-19 & 0.7479 & 0.1546 & $0.445-1.051$ & 4.837 & $<0.0001$ \\
Symptom associated with COVID-19 & 0.4713 & 0.1620 & $0.154-0.789$ & 2.909 & 0.0043 \\
Chronic Disease & 0.3310 & 0.1529 & $0.031-0.631$ & 2.165 & 0.0323 \\
[Dental Damage Stage]=0.2492+0.2680*[NDC]+0.0648*[NDI]+0.0565*[NRCT]- & \\
0.02105*[NTF]+0.0222*[NMT]+0.0212*[NC]+0.8486*[NHC]+0.5451*[SAC]+0.00801*[CD] \\
R squared: 0.6085, Multiple R: 0.7801, F: 24.8678 \\
\hline
\end{tabular}

NDC Number of dental caries, NDI Number of dental implants, NRCT Number of root canal treatment, $N T F$ Number of tooth filling, NMT Number of missing teeth, $N C$ Number of crown, $N H C$ Number of hospitalizations due to COVID-19, SAC Symptom associated with COVID-19, CD Chronic Disease

$\mathrm{R}$ squared value is the percentage of dental damage stage variance that can be explained by this model

\begin{tabular}{llllrr}
\hline Variable & Coefficient & SE & $95 \%$ CI & $t$ ratio & $P$ value \\
\hline Constant & 0.3705 & 0.1418 & $0.0927-0.6483$ & 2.614 & 0.0100 \\
Number of dental caries & 0.2715 & 0.0416 & $0.1899-0.3530$ & 6.525 & $<0.0001$ \\
Number of hospitalizations due to COVID-19 & 0.2700 & 0.1153 & $0.04398-0.4960$ & 2.341 & 0.0207 \\
Symptom associated with COVID-19 & 0.6604 & 0.1751 & $0.3172-1.004$ & 3.772 & 0.0002 \\
Chronic disease & 0.6667 & 0.1570 & $0.3590-0.9744$ & 4.247 & $<0.0001$ \\
[Dental Damage Stage] =0.3705+0.2715*[NDC]+0.2700*[NHC] $+0.6604 *[S A C]+0.6667 *[C D]$ & \\
R squared: 0.5499, Multiple R: 0.7139, F: 34.30 & & & &
\end{tabular}

$S E$ Standard error, $C I$ confidence interval, $N D C$ Number of dental caries, $N H C$ Number of hospitalizations due to COVID-19, SAC Symptom associated with COVID-19, CD: Chronic Disease

$\mathrm{R}$ squared value is the percentage of dental damage scale variance that can be explained by this model there was insignificant difference between the others. This suggests that those in the DD Stg 3 were seriously ill.

Table 2 presents the results of multiple regression analysis performed to estimate the effects of a number of independent variables, including NDC, NDI, NRCT, NTF, NMT, $\mathrm{NC}, \mathrm{NHC}, \mathrm{SAC}$ and $\mathrm{CD}$, on dental damage stage (dependent variable).

The model with nine independent variables in Table 2 was not considered appropriate $(p=0.1086)$. However, this model suggested that NDC, NHC, SAC and CD were effective alone on dental damage stage. Accordingly, the new model established using NDC, NHC, SAC and CD as independent variables to predict DD stage more accurately was considered appropriate $(p=0.0060)$ (Table 3$)$. This model suggested that DD stages were significantly affected by NDC, NHC, SAC and CD, whereby there was a close relationship between them. The correlational studies conducted to confirm the presence of this relationship revealed that DD stage had a positive high correlation with NDC and a

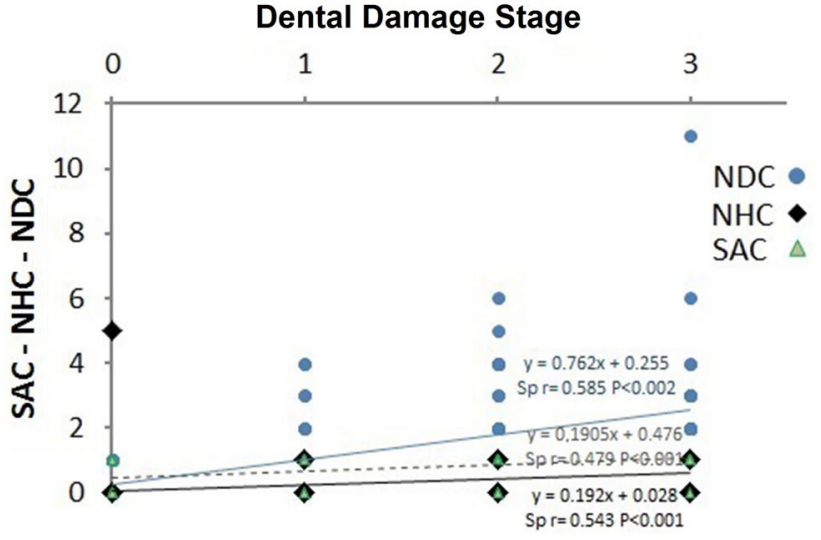

Fig. 4 There was a moderate positive relationship between the Dental Damage Stage, Number dental caries (NDC), Number of hospitalizations due to COVID-19 (NHC), Symptom associated with COVID-19 (SAC). $S p$ Spearman 
positive moderate correlation with $\mathrm{NHC}$ and SAC $(p>0.01)$ (Fig. 4). Similarly, there was a moderate positive correlation between DD Stg and CD (Spearman $r=0.4434,95 \%$ CI: 0.2932-0.5722; $p<0.0001)$.

Table 4 presents the incidence of symptoms and hospitalization rates due to COVID-19 according to DD Stg groups. Accordingly, $77 \%$ of the patients in all groups had at least one symptom of the COVID-19 disease. The most common symptom in all groups was cough (55\%), while the least one was diarrhea (8\%). All patients in the DD Stg 0 survived COVID-19 disease without hospitalization, while $75 \%$ of those in the DD Stg 3 were hospitalized due to the disease with an average length of hospital stay of 11.6 days.

In the study, it was determined that $59(43.1 \%)$ patients had A Rh (+), 29 (21.2\%) O Rh (+), 25 (18.2\%) B Rh (+), $14(10.2 \%)$ A Rh $(-), 4(2.9 \%)$ AB Rh $(+), 3(2.2 \%) \mathrm{O}$ Rh (-), 2 (1.5\%) B Rh (-) and 1 (0.7\%) AB Rh (-) blood group. Blood type did not present any characteristics among groups.

In addition, $28 \%$ of the COVID-19 patients had at least one chronic disease. Distribution of these diseases are as follows; chronic lung diseases (CLD) (chronic obstructive pulmonary disease, asthma) (28\%), hypertension (HT) (23\%), rheumatoid arthritis (RA) (13\%), chronic gastritis (CG) $(10 \%)$, hyperlipidemia (HL) $(8 \%)$, thyroid diseases (TD) (8\%), diabetes mellitus (DM) (5\%) and cancer (C) (5\%). Distribution of these diseases according to the groups was as follows; $2.5 \%$ in DD Stg $0,2.5 \%$ in DD Stg $1,44 \%$ in DD Stg 2 and $51 \%$ in DD Stg 3. There was only one chronic disease in Stg 0 (CLD) and Stg 1 (RA). In the DD Stg 2, there was $24 \%$ HT, $24 \%$ CG, $18 \%$ CLD, $12 \%$ RA, $12 \%$ TD, $5 \% \mathrm{DM}$ and $5 \% \mathrm{C}$, while in the DD Stg 3, there was 35\% CLD, 25\% HT, 15\% HL, 5\% C, 5\% DM and 5\% TD. Four of the patients had a second chronic disease. One of them was in the DD Stg 2 and three in the DD Stg 3. The rate of mortality was $75 \%$ for those with more than one chronic disease, and $8.5 \%$ for those with only one chronic disease.

\section{Discussion}

Dental caries and periodontal diseases are the most common chronic diseases caused by bacterial colonization (Biofilm) on the tooth surfaces, leading to tooth loss [18, 19]. Oral health affects general health, whereby cytokines and microbial products released in response to periapical and periodontal infections accelerate the development of systemic diseases by causing inflammation in distant organs [20-22]. Studies have reported that periodontal diseases accelerate the development of arteriosclerotic heart disease and diabetes [5, 23], Alzheimer's disease [24], and respiratory diseases [25]. Since periodontal diseases and endodontic apical periodontitis have similar microbiology, their negative effects on general health are also similar [26, 27].

This study used RBL classification as a symptom of periodontal disease, APGS classification as a symptom of periapical disease, and PPDC, which provides information about the pathophysiology of dental caries, as an indicator of oral hygiene. Using the DD Stg, which was developed based on these three markers, this study determined the patients' oral and dental health status. Periodontal status is evaluated using CAL, PPD and GR levels [11] and the amount of destruction in alveolar bone that support teeth radiologically [12]. RBL measurement can be used in cases, where it is not possible to perform CAL, PPD and GR clinically [10]. In previous studies, RBL has been generally calculated using a ruler, where the amount of lost or remaining bone was given as a percentage of the root or tooth length [12]. In recent years, RBL measurement on panoramic radiographs has been performed using methods that automatically detect bone loss
Table 4 COVID-19 symptoms and hospitalization rates according to dental damage stage groups

\begin{tabular}{llllll}
\hline & Stage 0 & Stage 1 & Stage 2 & Stage 3 & Total \\
\hline$n$ & 31 & 30 & 44 & 32 & 137 \\
Cough, $n(\%)$ & $7(23)$ & $18(60)$ & $28(64)$ & $23(72)$ & $76(55)$ \\
Fever, $n(\%)$ & $5(16)$ & $5(17)$ & $16(36)$ & $24(75)$ & $50(36)$ \\
Sore throat, $n(\%)$ & $1(3)$ & $3(10)$ & $20(45)$ & $17(53)$ & $41(30)$ \\
Chest pain $n(\%)$ & $2(6)$ & $2(7)$ & $9(20)$ & $10(31)$ & $23(17)$ \\
Joint pain, $n(\%)$ & $1(3)$ & $7(23)$ & $11(25)$ & $18(56)$ & $37(27)$ \\
Back pain, $n(\%)$ & $3(10)$ & $7(23)$ & $6(14)$ & $6(19)$ & $22(16)$ \\
Weakness, $n(\%)$ & $5(16)$ & $9(30)$ & $14(32)$ & $17(53)$ & $45(33)$ \\
Diarrhea, $n(\%)$ & $1(3)$ & $2(7)$ & $3(7)$ & $5(16)$ & $11(8)$ \\
Dyspnoea, $n(\%)$ & $1(3)$ & $3(10)$ & $15(34)$ & $21(66)$ & $40(29)$ \\
Loss of smell and taste, $n(\%)$ & $1(3)$ & $2(7)$ & $4(9)$ & $8(25)$ & $15(11)$ \\
Hospitalization, $n(\%)$ & $0(0)$ & $2(7)$ & $13(30)$ & $24(75)$ & $39(28)$ \\
Average length of hospital stay, day & 0 & 4 & 11.8 & 11.6 & 11.3 \\
\hline
\end{tabular}

$n$ Number 
such as CAD based on deep learning and the convolution neural network (CNN) [16, 17, 28]. We successfully used the APGS classification, which we created based on the PAI, in the evaluation of AP in our previous studies [7, 8]. This classification was also compatible with the markers associated with inflammatory process (IL-6, hsCRP and PAPP-A).

This study found that the groups with high dental damage had higher CD and NDC, and that the NMT value was quite different in the group with the most severe dental damage. These results suggest that the DD Stg system based on APGS, RBL and PPDC can be used as an important indicator of dental health. In the past, many similar scales and scoring have been developed as an indicator of oral and dental status [29]. The most used ones are the Total Dental Index [30], the Modified Total Dental Index [31, 32], the Dental Asymptotic Score [33], the Brief Oral Health Status Examination, and the Global Oral Health Scale. All of these classifications require intraoral examination and measurements. However, today, there is a need for classifications to show dental health status in a more practical and comprehensive way. The classification developed in this study, which was planned to achieve this aim, was based on more rational radiographic data. Therefore, this classification does not require a direct contact with patients during the COVID19 pandemic, and has a practical and easy algorithm that provides broader information about dental health status. Since the study group had a risk of COVID-19 transmission, the study was conducted retrospectively using radiographs and dental records. Therefore, it did not include intraoral examination of the patients. We think that this classification will be useful in determining the oral and dental health status in patients with infectious diseases such as COVID19. According to the classification developed in this study, there was a significant correlation between the DD Stg and the morbidity and mortality of COVID-19 disease. Namely, the rate of mortality and chronic disease due to COVID-19 was significantly higher in patients in the DD Stg 2 and 3. This result is consistent with those in studies reporting that the rate of mortality due to COVID-19 disease is higher in patients with chronic diseases [34, 35]. In addition, the presence of a relationship between immune system weakness and morbidity/mortality due to COVID-19 disease [36, 37] suggests that there may be an indirect relationship between DD Stg and immune system.

Oral mucosa is a potential entry route for the SARSCoV-2 virus that causes COVID-19 disease [38]. The presence of ACE2, which is the cellular entry receptor of SARSCoV-2, in oral mucosa tissues, tongue and gum epithelium supports that oral cavity is an entry point of the virus [39]. There may be an increase in ACE2 receptors in people with poor oral health $[39,40]$. This is why oral hygiene and dental health status are important in combating the COVID-19 pandemic. Therefore, oropharyngeal colonization of viruses and bacteria can be prevented and the risk of respiratory complications due to these pathogens can be reduced by improving oral health. The fact that improving oral health reduces both occurrence and progression of respiratory tract diseases especially in the elderly population and intensive care patients [41] supports this view.

This study found that patients in the DD Stg 3, who had the worst dental indicators, had higher mean age compared to the others. This result suggests that those with poor dental status were mostly from the elderly population. This was actually an expected result. Because immune system weakens and general body functions deteriorate by aging. Parallel to this, a deterioration in oral and dental health is also expected. Moreover, numerical and dimensional increase in dental caries occurs due to the net mineral loss as a result of increased demineralization, decreased remineralization, insufficiency in bacterial defense and chronic malnutrition along with aging. In addition, the balanced population of oral microorganisms deteriorates due to aging, changing into a population that produces acid or survives in an acid environment [42, 43]. The elderly population is also at risk of developing serious complications related to COVID-19 $[44,45]$. All these explain why patients in the DD Stg 3 with poor dental health were mostly the elderly. Therefore, it is important to pay special attention to the population aged 50 years and above in terms of preserving oral and dental health. Because oral and dental health is directly related to the maintenance of healthy nutrition. Nutrition, on the other hand, is a vital activity related to both the supply of all mineral, micro and macro substances for physical regeneration and the immune system $[46,47]$. The fact that patients in the DD Stg 3 were older and had higher rate of mortality due to COVID-19 disease confirms these evaluations.

Higher rates of hospitalization in patients with higher DD Stg suggest a correlation between the stage of dental damage and the severity of COVID-19 disease. This may be because individual resistance to viral diseases such as COVID-19 is dependent on general health status, including both immune system and dental health status. Because Scannapieco et al. reported a relationship between infectious respiratory diseases and oral hygiene [41]. The present study also found that COVID-19 symptoms were more observed in those with higher dental damage and the mortality rate was very higher in patients with more severe dental damage (e.g., those in the DD Stg 3), which also supports these researchers. However, it should not be forgotten that dental status, which can be an indicator of oral hygiene, is also associated with behavioral conditions, in other words, it is multi-factorial. Thus, oral hygiene and dental status may also be indicators of resistance to viral diseases such as COVID-19, in relation to general health status.

All independent variables (NHC, SAC and CD) except for NDC, which were created as an expression of the dental 
health status and were found to affect the prediction of DD staging, were parameters related to the course of COVID19 disease. The reason why these parameters are related to dental health status can be explained by the pathophysiological mechanism of COVID-19 disease. That is to say, not only the local exposure of the teeth lies behind dental diseases. In a wider scope, immunological mechanisms involving the body's defense system against viruses and bacteria, biochemical reactions in regeneration or remineralization involving cofactors such as calcium, phosphate, ferrous, magnesium, organic (vitamins) or metalloorganic complexes and macronutrients, and physiological processes involving both macro and microcirculatory system that enable fluid and nutrient exchange can play a role in the development of dental diseases [48-50]. These parameters are also related to chronic diseases, hospitalization time, and disease symptoms.

\section{Conclusion}

The relationship detected between DD stage and the severity and prognosis of viral disease such as COVID-19 was found to be remerkable for extensive studies.

\section{Compliance with ethical standards}

Conflict of interest There is no authors potential conflict of interest in this paper.

Ethical approval For conducting the study, an ethical approval was obtained from the University of Health Sciences, Hamidiye Clinical Research Ethics Committee (registration number: 20/180). Furthermore, this study was conducted with the approval (No. 2020-05-27T1220-31) of the T.R Ministry of Health Research Platform.

Human or animal rights This article does not contain any studies with human or animal subjects performed by the any of the authors.

Informed consent Additional informed consent was obtained from all patients for which identifying information is included in this article.

\section{References}

1. Ren YF, Rasubala L, Malmstrom H, Eliav E. Dental care and oral health under the clouds of COVID-19. JDR Clin Trans Res. 2020;5(3):202-10.

2. Liu Y-C, Kuo RL, Shih S-R. COVID-19: the first documented coronavirus pandemic in history. Biomed J. 2020. https://doi. org/10.1016/j.bj.2020.04.007.

3. Lu R, Zhao X, Li J, et al. Genomic characterisation and epidemiology of 2019 novel coronavirus: implications for virus origins and receptor binding. Lancet. 2020;395(10224):565-74.
4. Botros N, Iyer P, Ojcius DM. Is there an association between oral health and severity of COVID-19 complications? Biomed J. 2020. https://doi.org/10.1016/j.bj.2020.05.016.

5. Khumaedi AI, Purnamasari D, Wijaya IP, et al. The relationship of diabetes, periodontitis and cardiovascular disease. Diabetes MetabSyndClin Res Rev. 2019;13(2):1675-8.

6. Ørstavik D, Kerekes K, Eriksen HM. The periapical index: a scoring system for radiographic assessment of apical periodontitis. Dent Trauma. 1986;2(1):20-34.

7. Sirin DA, Ozcelik F, Ersahan S, et al. The importance of inflammatory biomarkers, IL- 6 and PAPP-A, in the evaluation of asymptomatic apical periodontitis. Odontology. 2020. https://doi. org/10.1007/s10266-020-00534-8.

8. Sirin DA, Ozcelik F, Uzun C, et al. Association between C-reactive protein, neutrophil to lymphocyte ratio and the burden of apical periodontitis: a case-control study. Acta Odon Scand. 2019;77(2):142-9.

9. Caton JG, Armitage G, Berglundh T, et al. A new classification scheme for periodontal and peri-implant diseases and conditionsIntroduction and key changes from the 1999 classification. J Periodontol. 2018;89:1-8.

10. Tonetti MS, Greenwell H, Kornman KS. Staging and grading of periodontitis: framework and proposal of a new classification and case definition. J Periodontol. 2018;89:159-72.

11. Keagle JG, Garnick JJ, Searle JR, et al. Gingival resistance to probing forces: I determination of optimal probe diameter. J Periodontol. 1989;60(4):167-71.

12. Papapanou PN, Lindhe J. Epidemiology of periodontal diseases. In: Lindhe J, Karring T, Lang NP, editors. Clinical Periodontology and Implant Dentistry. Oxford: Blackwell; 2003. p. 50-73.

13. Chang HJ, Lee SJ, Yong TH, et al. Deep learning hybrid method to automatically diagnose periodontal bone loss and stage periodontitis. Sci Rep. 2020;10(1):7531.

14. Conrads G, About I. Pathophysiology of dental caries. In: Schwendicke F, Frencken J, Innes N, editors. Caries excavation: Evolution of treating cavitated carious lesions. Monographs in Oral Science. Basel: Karger Publishers; 2018. p. 1-10.

15. Anusavice KJ. Management of dental caries as a chronic infectious disease. J Dent Educ. 1998;62(10):791-802.

16. Krois J, Ekert T, Meinhold L, et al. Deep learning for the radiographic detection of periodontal bone loss. Sci Rep. 2019;9(1):8495.

17. Kim J, Lee H-S, Song I-S, et al. DeNTNet: deep neural transfer network for the detection of periodontal bone loss using panoramic dental radiographs. Sci Rep. 2019;9(1):17615.

18. Albandar JM. Epidemiology and risk factors of periodontal diseases. Dental Clinics. 2005;49(3):517-32.

19. Albandar JM, Brunelle JA, Kingman A. Destructive periodontal disease in adults 30 years of age and older in the United States, 1988-1994. J Periodontol. 1999;70(1):13-29.

20. Bui FQ, Almeida-da-Silva CLC, Huynh B, et al. Association between periodontal pathogens and systemic disease. Biomed J. 2019;42(1):27-35.

21. Weidlich P, Cimões R, Pannuti CM, et al. Association between periodontal diseases and systemic diseases. Braz Oral Res. 2008;22:32-43.

22. Paquette DW. The periodontal infection-systemic disease link: a review of the truth or myth. J InternatAcadPeriodontol. 2002;4(3):101-9.

23. Jepsen S, Stadlinger B, Terheyden H, et al. Guest editorial science transfer: oral health and general health-the links between periodontitis, atherosclerosis, and diabetes. J Clin Periodontol. 2016;42(12):1071-3.

24. Wu Z, Nakanishi H. Connection between periodontitis and Alzheimer's disease: possible roles of microglia and leptomeningeal cells. J Pharmacol Sci. 2014;126(1):8-13. 
25. Zhou X, Wang Z, Song Y, et al. Periodontal health and quality of life in patients with chronic obstructive pulmonary disease. Respir Med. 2011;105(1):67-73.

26. Rupf S, Kannengiesser S, Merte K, et al. Comparison of profiles of key periodontal pathogens in periodontium and endodontium. Dent Traumatol. 2000;16(6):269-75.

27. Kerekes K, Olsen I. Similarities in the microfloras of root canals and deep periodontal pockets. Dent Traumatol. 1990;6(1):1-5.

28. Schmidhuber J. Deep learning in neural networks: an overview. Neural Networks. 2015;61:85-117.

29. Relvas M, Diz P, Seoane J, et al. Oral health scales: design of an oral health scale of infectious potential. Med Oral Patol Oral Cir Bucal. 2013;18(4):664.

30. Mattila KJ, Nieminen MS, Valtonen VV, et al. Association between dental health and acute myocardial infarction. Br Med J. 1989;298:779-81.

31. Beck J, Garcia R, Heiss G, et al. Periodontal disease and cardiovascular disease. J Periodontol. 1996;67(10):1123-37.

32. Mattila KJ. Dental infections as a risk factor for acute myocardial infarction. Eur Heart J. 1993;14:51-3.

33. Janket SJ, Qvarnström M, Meurman JH, et al. Asymptotic dental score and prevalent coronary heart disease. Circulation. 2004;109(9):1095-100.

34. Guo T, Fan Y, Chen M, et al. Cardiovascular implications of fatal outcomes of patients with coronavirus disease 2019 (COVID-19). JAMA Cardiol. 2020;5(7):1-8.

35. Murthy S, Gomersall CD, Fowler RA. Care for critically ill patients with COVID-19. JAMA. 2020;323(15):1499-500.

36. Pedersen SF, Ho YC. SARS-CoV-2: a storm is raging. J Clin Invest. 2020;130(5):2202-5.

37. Zhang $\mathrm{C}, \mathrm{Wu} \mathrm{Z}$, Li JW, et al. The cytokine release syndrome (CRS) of severe COVID-19 and Interleukin-6 receptor (IL-6R) antagonist Tocilizumab may be the key to reduce the mortality. Int J Antimicrob Agents. 2020;55(5): 105954.

38. Peng X, Xu X, Li Y, et al. Transmission routes of 2019-nCoV and controls in dental practice. Int J Oral Sci. 2020;12(1):9.

39. Xu H, Zhong L, Deng J, et al. High expression of ACE2 receptor of 2019-nCoV on the epithelial cells of oral mucosa. Int J Oral Sci. 2020;12(1):8.
40. Chen L, Zhao J, Peng J, et al. Detection of 2019-nCoV in saliva and characterization of oral symptoms in COVID-19 patients. SSRN. 2020. https://doi.org/10.2139/ssrn.3556665.

41. Azarpazhooh A, Leake JL. Systematic review of the association between respiratory diseases and oral health. J Periodontol. 2006;77(9):1465-82.

42. Mjör IA. Age changes in the teeth. In: Holm-Pedersen P, Löe $\mathrm{H}$, editors. Geriatric dentistry: A textbook of oral gerontology. Copenhagen: Munksgaard; 1986. p. 94-101.

43. Niessen LC, Jones JA. Oral health changes in the elderly: Their relationship to nutrition. Postgraduate Med. 1984;75(5):231-7.

44. Boccardi V, Ruggiero C, Mecocci P. COVID-19: A geriatric emergency. Geriatrics. 2020;5(2):24.

45. Swiss Academy of Medical Sciences. COVID-19 pandemic: Triage for intensive-care treatment under resource scarcity. Swiss Med Wkly. 2020;150:20229.

46. Chandra RK. Nutrition and the immune system: an introduction. Am J Clin Nutr. 1997;66(2):460-3.

47. Childs CE, Calder PC, Miles EA. Diet and immune function. Nutrients. 2019;11(8):1933.

48. Abou Neel EA, Aljabo A, Strange A, et al. Demineralizationremineralization dynamics in teeth and bone. Int $\mathrm{J}$ Nanomed. 2016;11:4743-63.

49. González-Cabezas C, Fernández CE. Recent advances in remineralization therapies for caries lesions. Adv Dent Res. 2018;29(1):55-9.

50. Marton IJ. The influence of chronic apical periodontitis on oral and general health. Fogorv Sz. 2007;100(5):200-9.

Publisher's Note Springer Nature remains neutral with regard to jurisdictional claims in published maps and institutional affiliations. 